\title{
A Unified Theory of Arcs and their Electrodes
}

\author{
J.J. Lowke
}

CSIRO Telecommunications and Industrial Physics, Box 218, Lindfield, Sydney, NSW 2070, Australia

\begin{abstract}
For arcs at atmospheric pressure which have cathodes that are thermionic emitters, it is possible to calculate the major properties of the arc and the electrodes as a function of current, by accounting for electrode shape and the heat transfer processes occurring at the surface of the electrodes. Such processes occur due to electron and ion emission and absorption and also from radiation emission and absorption. Electrical resistance of the plasma near the electrodes is calculated either by taking account of ambipolar diffusion or simply using the local plasma value, with mesh sizes sufficiently large to account for ambipolar diffusion. Derived temperature profiles are in fair agreement with experiment. Results of electrode temperatures and arc melting effects, including such phenomena as the transition from globular to spray modes in arc welding, are also in good agreement with experiment. Prediction of properties for non-thermionic cathodes still constitute a major problem. Approximate calculations indicate that electrons at the surface of the non-thermionic cathodes may be produced by photo ionisation of neutral atoms rather than by field emission.
\end{abstract}

\section{INTRODUCTION}

Properties of electric arcs at atmospheric pressure depend not only on the arc plasma medium, but also on the electrodes. In particular they depend on the electrode shape and whether or not the cathode is a thermionic emitter. With the availability of modern high speed computers, it has recently become possible to make detailed predictions of properties of electric arcs from basic material properties, provided the arcs have thermionically emitting cathodes. Thus, given the electrode shape and the material properties of the plasma and the electrodes, it is possible to calculate the maximum temperature of the arc, the diameter of the arc, whether the electrodes will melt and the velocity profiles of the plasma flow within the arc, for any given arc current.

To make this type of prediction, we need to understand the physical processes that are occurring. We also need to know which of these processes are dominant, so that we can make adequate approximations to be able to make computations in a reasonable computation time. It is often the case that we understand the many atomic processes very well, but even with the enormous improvements in the speed and availability of computers, we still are unable to make economic computations in a reasonable time. The art of making valid approximations is then all important.

The major requirement is to include an account of the electrode shape [1] and the heat transfer processes that are occurring at the surface of the electrodes. For thermionic cathodes, the non equilibrium electrical conductivity of the plasma near the cathode surface can be obtained by calculating charge densities taking into account ambipolar diffusion [2]. Calculations indicate that for arcs with thermionic cathodes, the regions where positive and negative charges are unequal are small and can be neglected [3].

The calculation of detailed properties of arcs with non thermionic cathodes represents a major unsolved problem. Experimental evidence indicates that there is a sheath at the surface of cathodes with a voltage drop of 15-20 V, which has a remarkably, small dependence on cathode material, plasma composition or arc current ; Kesaev [4], Matsumura et al [5]. There must be some mechanism for the production of the electrons for the arc current, at, or in front of the cathode. For vacuum arcs, the electron emission is generally accepted as being due to field emission caused by a high field produced by a sheath of positive ions in front of the cathode [6]. For high pressure arcs there is another possibility - photo-ionisation in the cathode sheath of neutral atoms in front of the cathode by ultra-violet photons from ions in the plasma. 
We discuss the arc column in section 2 , the cathode and cathode sheath for thermionic cathodes in Section 3, the anode and anode sheath in Section 4, and the cathode and cathode sheath for nonthermionic cathodes in Section 5. Finally, in Section 6, we present specific predictions compared with experiment of (a) the tips of sharp tungsten cathodes melting off at high current, (b) the effect on arc and electrode properties of adding hydrogen to argon, (c) the change from globular to spray transfer of molten metal in "Gas Metal Arc Welding (GMAW)" for arcs in argon, and (d) the different properties of electrode melting for arcs in carbon dioxide.

\section{ARC COLUMN}

It has been known for a long time that the column of electric arcs can be well represented as a plasma in local thermodynamic equilibrium; Finkenburg and Maecker [7]. The basic equations defining the temperature are conceptually simple, namely the equations for conservation of mass, energy, momentum and current. The equation for conservation of mass is

$$
\frac{\partial \rho}{\partial t}+\frac{1}{r} \frac{\partial}{\partial r}\left(r \rho u_{r}\right)+\frac{\partial}{\partial z}\left(\rho u_{z}\right)=0
$$

$\rho$ is the density, and $u_{r}$ and $u_{z}$ are the radial and axial velocities in the $r$ and $z$ directions. The equation for conservation of energy is

$$
\frac{\partial(\rho \mathrm{h})}{\partial t}+\frac{1}{\mathrm{r}} \frac{\partial}{\partial \mathrm{r}}\left(\mathrm{r} \rho \mathrm{u}_{\mathrm{r}} \mathrm{h}\right)+\frac{\partial}{\partial \mathrm{z}}\left(\rho \mathrm{u}_{\mathrm{z}} \mathrm{h}\right)=\frac{1}{\mathrm{r}} \frac{\partial}{\partial \mathrm{r}}\left(\frac{\mathrm{rk}}{\mathrm{c}_{\mathrm{p}}} \frac{\partial \mathrm{h}}{\partial \mathrm{r}}\right)+\frac{\partial}{\partial z}\left(\frac{\mathrm{k}}{\mathrm{c}_{\mathrm{p}}} \frac{\partial \mathrm{h}}{\partial \mathrm{z}}\right)+\frac{\mathrm{j}^{2}+\mathrm{jz}^{2}}{\sigma}-\mathrm{U}
$$

$h$ is the enthalpy, $k$ is the thermal conductivity, $U$ the radiation emission coefficient, $c_{p}$ the specific heat, $\sigma$ the electrical conductivity, and $\mathrm{j}_{\mathrm{r}}$ and $\mathrm{j}_{\mathrm{z}}$ the radial and axial current densities. The equations for the conservation of radial and axial momentum are

$$
\begin{gathered}
\frac{\partial\left(\rho u_{r}\right)}{\partial t}+\frac{1}{r} \frac{\partial}{\partial r}\left(r \rho u_{r}^{2}\right)+\frac{\partial}{\partial z}\left(\rho u_{r} u_{z}\right)=-\frac{\partial P}{\partial r}-j_{z} B_{\theta}+\frac{1}{r} \frac{\partial}{\partial r}\left(2 r \mu \frac{\partial u_{r}}{\partial r}\right) \\
+\frac{\partial}{\partial z}\left(\frac{\mu \partial u_{z}}{\partial r}+\frac{\mu \partial u_{r}}{\partial z}\right)-2 \mu \frac{u_{r}}{r^{2}}
\end{gathered}
$$

and

$$
\begin{gathered}
\frac{\partial\left(\rho u_{z}\right)}{\partial t}+\frac{1}{r} \frac{\partial}{\partial r}\left(r \rho u_{z} u_{r}\right)+\frac{\partial}{\partial z}\left(\rho u_{z}^{2}\right)=-\frac{\partial p}{\partial z}+j_{r} B_{\theta}+\frac{\partial}{\partial z}\left(2 \mu \frac{\partial u_{z}}{\partial z}\right) \\
+\frac{1}{r} \frac{\partial}{\partial r}\left(\frac{r \mu \partial u_{z}}{\partial r}+\frac{r \mu \partial u_{r}}{\partial z}\right)+\rho g
\end{gathered}
$$

$P$ is the pressure, $\mu$ is the viscosity, $g$ the gravitational acceleration and $B_{\theta}$ the azimuthal magnetic field. $B_{\theta}$ is determined from Maxwell's equation

$$
\frac{1}{\mathrm{r}} \frac{\partial}{\partial \mathrm{r}}(\mathrm{rB} \theta)=\mu_{0} j_{z}
$$

where $\mu_{0}$ is the permeability of free space; $\mathbf{j}=\sigma \mathrm{E}$, where $\mathbf{E}=-\nabla \mathrm{V}$ and $\mathrm{V}$ is the potential. The current continuity equation is

$$
\frac{1}{\mathrm{r}} \frac{\partial}{\partial \mathrm{r}}\left(\mathrm{r} \sigma \frac{\partial \mathrm{V}}{\partial \mathrm{r}}\right)+\frac{\partial}{\partial \mathrm{z}}\left(\sigma \frac{\partial \mathrm{V}}{\partial \mathrm{z}}\right)=0
$$


The above constrvation equations also apply to the solid electrodes, and the molten metal, if the electrodes are molten, as is the case for welding arcs, discussed later in Section 6.3 and 6.4. Then the boundaries of these three phases need to be specified on the grid system and the material functions at each grid point chosen appropriate to each phase and the local temperature.

\section{THERMIONIC CATHODES AND CATHODE SHEATHS}

\subsection{Electrode Heat Exchange Processes}

Calculations at points on the electrode surface need to include the heat transfer processes occurring at the surfaces. Additional to the usual energy fluxes by thermal conduction are energy losses by thermal radiation from the hot electrode, cooling or heating by the electrons leaving or entering the solid and heating by ion bombardment. Heating of the electrodes by radiation from the arc is neglected. For the cathode, the additional energy flux, $F$, is

$$
F=-\varepsilon a T^{4}-\left|j_{e}\right| \phi+\left|j_{i}\right| V_{i}
$$

$\varepsilon$ is the emissivity of the surface, $T$ is the surface temperature, $\phi$ is the work function, $\mathrm{j}_{\mathrm{e}}$ and $\mathrm{j}_{\mathrm{i}}$ are the electron and ion current density, $a$ is the Stefan Boltzmann constant, and $V_{\mathbf{i}}$ is the ionisation potential of the plasma. The term in $\phi$ represents the loss in energy from electrons leaving the cathode and overcoming the work function potential. The ion current density, $j_{i}$, is taken to be $j-j_{R}$ at the cathode surface, where $j$ is the cathode surface current density from the current continuity equation (6) and $j_{R}$ is the theoretical electron current due to thermionic emission obtained from $j_{R}=A T^{2} \exp (-\phi e / k T)$; e is the electronic charge, $\mathrm{k}$ is Boltzmann's constant and $\mathrm{A}$ is the thermionic emission constant for the surface of the cathode. If $j_{R}$ is greater than $j$ we take $j_{j}$ to be zero. The assumption that heating of the cathode is given by $\left|j_{i}\right| V_{i}$ is supported by the experimental results of Winters et al [8]. These experiments suggest that the full ionisation potential $V_{i}$ is delivered to the cathode, there being no reduction in $V_{i}$ by $\phi$, presumably because the electron for neutralisation of the ion is provided within the cathode surface.

\subsection{Cathode-Arc Sheath Resistance}

There is a major problem associated with electrode effects. We need to decide how to calculate the electrical resistance of the plasma near the electrodes, where the plasma temperatures are relatively low. If we assume local thermodynamic equilibrium and a collision dominated sheath, the electron density would be effectively zero at the melting temperature of the electrodes, so that then the equilibrium electrical conductivity would be zero. For finite electrical conduction, there must be some mechanism such as thermionic emission or ambipolar diffusion to make these regions highly conducting. To account for ambipolar diffusion an effective electrical conductivity has been calculated for mesh intervals at the cathode surfaces [2,9-11].

First, the electron continuity equation is solved for the electrode sheath mesh interval ie

$$
\nabla .\left(D_{A} \nabla n_{e}\right)+S-m_{e}{ }^{2}=0
$$

$\mathrm{D}_{\mathrm{A}}$ is the ambipolar diffusion coefficient [10] for the local temperature given by $2 \mathrm{kT} \mu_{\mathrm{i}} / \mathrm{e}, \mu_{\mathrm{i}}$ is the ion mobility, $\mathrm{n}_{\mathrm{e}}$ is the electron number density and $\gamma$ is the electron-on recombination coefficient. A source term, $S$, representing the production of electrons and ions by thermal ionisation, is defined as $S=\gamma n_{e q}{ }^{2}$, where $n_{\mathrm{eq}}$ is the equilibrium plasma value of electron density for the local plasma temperature. Then solutions for $n_{e}$ in the sheath will be the equilibrium values if there is no diffusion. For the solution of equation (8), boundary conditions are needed for $n_{e}$. On the plasma side of the mesh interval the equilibrium plasma value is used. On the electrode side we take $n_{e}=j_{R} / e v_{t}$, where the thermal velocity $v_{t}$ is obtained from $\mathrm{mv}_{\mathrm{t}}{ }^{2} / 2=2 \mathrm{kT}$; $\mathrm{m}$ is electron mass.

Second, using the values of electron density as a function of distance within the sheath, the effective electrical conductivity of the sheath, $\sigma_{\text {eff }}$, is derived. A generalised form of Ohm's Law [9] is used: 


$$
j=\frac{n_{e} e}{\left\{n_{0} / n_{T} \mu_{e} E+2 e n_{e} n_{e q}(T) / n_{T} \sigma E\right\}}
$$

$n_{0}$ is the equilibrium neutral particle density, $\mu_{e}$ is the electron mobility and $n_{T}=n_{0}+2 n_{e}$ is the total particle density. This expression reduces to $j=\sigma E$ in the plasma where $n_{0}=0$ and to $j=n_{e} e \mu_{e} E$ in a cold gas where $n_{0} \sim n_{T}$ and $n_{e q} \sim 0$. Equation (9) is used to derive $E$ as a function of position within the sheath for a given $j$ and thus, by integration, the potential drop, $V_{s}$, across the mesh interval is obtained. Then $\sigma_{\text {eff }}=\mathrm{js} / \mathrm{V}_{\mathrm{S}}$ where $\mathrm{s}$ is the mesh thickness.

Equation (9) is a general approximation that can be derived for a partially ionised plasma. We take $\mathrm{j}$ $\sim \mathrm{je}_{\mathrm{e}}$, where the electron current density is given by $\mathrm{je}_{\mathrm{e}}=\mathrm{n}_{\mathrm{e}} \mathrm{eW}$, where the drift velocity, $\mathrm{W}$, of the elctrons in a mixture of neutrals and charged particles can be represented to a good approximation by $\mathrm{W}$ $=E e / m v$ [12]. The total collision frequency, $v$, is given by $v=v_{n}+v_{c}$, the sum of the electron collision frequencies, $v_{\mathrm{n}}$, with neutral particles and, $v_{\mathrm{c}}$, with charged particles. Using $\mathrm{W}_{\mathbf{n}}=\mathrm{Ee} / \mathrm{m} v_{\mathrm{n}}$, it follows that $v_{n}$ is given by $e_{\mathrm{O}} / \mathrm{m}_{\mathrm{e}} \mathrm{n}_{\mathrm{T}}$, where $\mathrm{W}_{\mathrm{n}}=\mu_{\mathrm{e}} \mathrm{E}$ is the drift velocity of electrons in neutral atoms, and we have weighted this collision frequency with the proportion of neutral atoms, $n_{\mathrm{O}} / \mathrm{n}_{\mathrm{T}}$. Similarly, $v_{c}$ is given by $n_{e} e^{2} 2 n_{e q} / m \sigma n_{T}$, where we have used (a) the relationship between the drift velocity, $W_{c}$, of electrons in an equilibrium plasma and $\sigma$, of $n_{e} e W_{c}=\sigma E$, (b) $W_{c}=E e / m v_{c}$ and (c) a weighting factor of $2 \mathrm{n}_{\mathrm{eq}} / \mathrm{n}_{\mathrm{T}}$ for the proportion of charged particles.

Figure 1 shows an example of a numerical solution of equation (8) for a region of the plasma at the tip of the cathode for a $200 \mathrm{~A}$ arc. The charge densities near the cathode, indicated by open circles, are orders of magnitude above the equilibrium values, labelled LTE, due to the influence of ambipolar diffusion. It is seen that in the main plasma the influence of diffusion is small, but that there is a region of significant non-equilibrium near the cathode surface which extends over a distance of about $0.005 \mathrm{~cm}$. To obtain solutions of equation (8), values of $\gamma$ are required as a function of temperature and charge density. We have used the classical formula $\gamma=1.1 \times 10^{-12} \mathrm{ne}_{\mathrm{e}} \mathrm{T}^{-4.5} \mathrm{~cm}^{3} \mathrm{~s}^{-1}$ of Hinnov and Hirschberg [13], discussed by Mitchner and Kruger [14]. Other values are given by Biberman et al [15] and Hoffert and Lien [16].

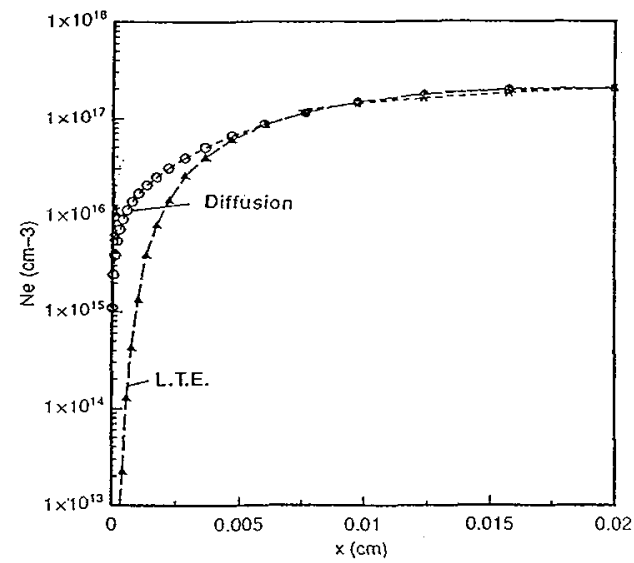

Fig. 1 Non-equilibrium charge densities at cathode for $200 \mathrm{~A}[11]$.

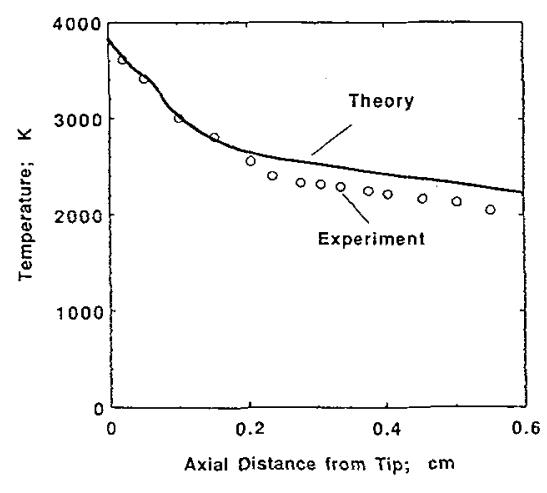

Fig. 3 Calculated [11] and experimental [18] cathode surface temperatures.

Equations (1-6) can be solved for any given arc current $[2,10,11]$ to obtain $h, P, u_{r}, u_{z}, B_{\theta}$ and $V$ as a function of $r$ and $z$. Temperature is obtained through the temperature dependence of $h$. The material 
functions $\rho, c_{p}, k, \sigma, U, \varepsilon, \phi, V_{i}, n_{e q}, n_{0}$, and $\gamma$ are required input as a function of temperature, from, for example, Murphy and Arundell [17]. For $\rho, c_{p}, k$ and $\sigma$, values are required for the plasma and the electrodes, for both the solid and liquid state.

Figure 2 shows predicted temperature contours for a $200 \mathrm{~A}$ arc in argon with a thoriated tungsten cathode of 60 degree angle, separated from an anode of copper by $5 \mathrm{~mm}[10,11]$. The bottom face of the copper is water cooled to maintain it at room temperature, assumed to be $300 \mathrm{~K}$. Experimental values of the arc temperature from Haddad and Farmer [18] are shown as points. Figure 3 shows theoretical values of temperature of the surface of the tungsten cathode taken from Figure 2, as a function of distance from the cathode tip. Also shown are experimental values of surface temperature, taken spectroscopically, and shown as points [19]. For both figures the agreement between theory and experiment is very satisfactory. Results are largely independent of mesh size for mesh sizes varying from 0.003 to $0.02 \mathrm{~cm}[11]$.

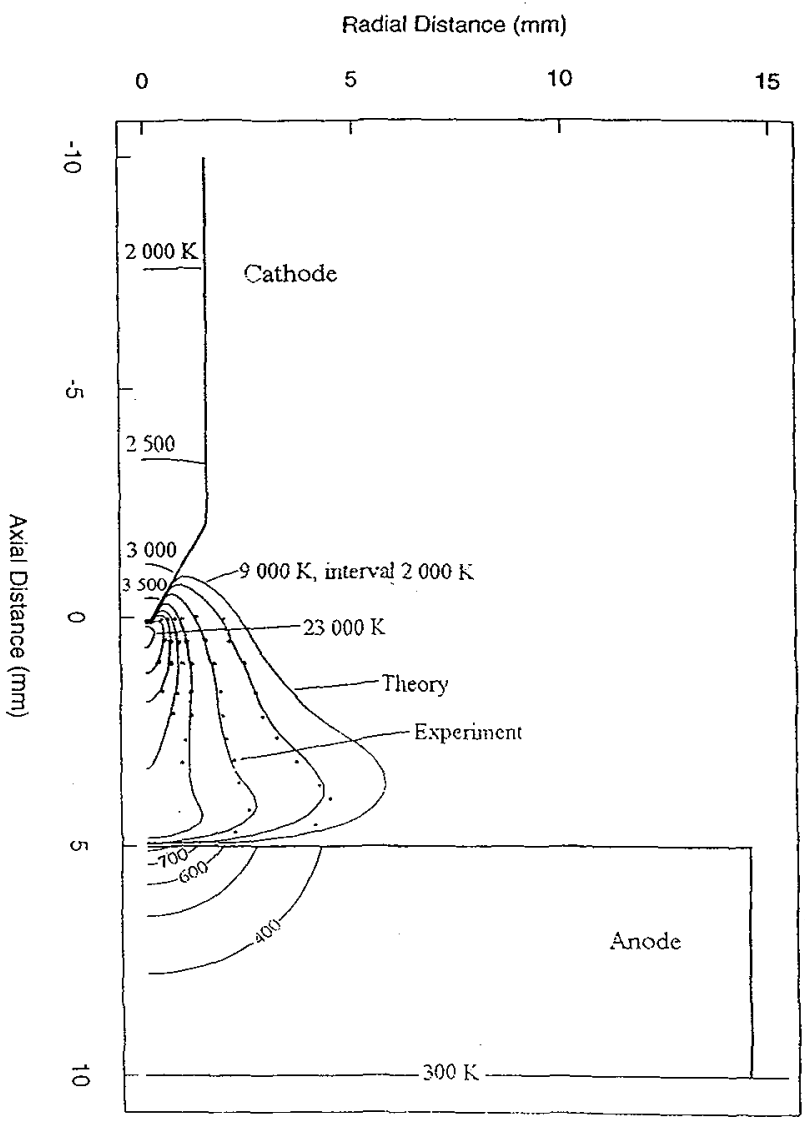

Fig. 2 Predicted temperature contours for a $200 \mathrm{~A}$ arc [11], compared with experiment [18].

\section{ANODES AND ANODE SHEATHS}

At the anode surface we change the sign of the term in $\phi$ of equation (7) as electrons heat the anode due to the work function potential. We assume that there is no ion current and hence no ion heating at the anode. For the anode it is not possible to calculate the resistance of the anode sheath by using the same ambipolar diffusion treatment as used for the cathode. It is usual to set the charged particle densities to zero as a boundary condition at any absorbing anode surface. However, the calculated electric field at 
the anode surface with $n_{e}=0$ from equation (9) would be infinite. If current due to diffusion is included in the analysis, it is found that because of the large density gradients, the diffusion current is so large that the electric field immediately in front of the anode reverses sign. This effect has been found by Dinulescu and Pfender [20]. Our use of $j=\sigma E$ in the main iteration algorithm requires a negative value of $\sigma$ if there is a reversal in the sign of $E$ to maintain positive current. However, our method for the solution of the current continuity equation (6) is unstable for negative values of $\sigma$.

Thus in our solutions of the equations we have omitted sheath effects at the anode and simply taken the local values of the electrical conductivity near the anode. Numerical solutions are a function of the grid spacing at the anode, as can be understood by considering that in the limit of a very fine mesh at the anode, the plasma temperature would approach that of the anode, and the electrical conductivity would approach zero. The calculated current density at the anode as a function of the mesh size in the $z$ direction at the anode for a $200 \mathrm{~A}$ arc gives agreement with the experimental results of Nestor [21] if the mesh size near the anode is about $0.05 \mathrm{~cm}$. This mesh size corresponds to an ambipolar sheath thickness of $0.05 \mathrm{~cm} \mathrm{[11]} \mathrm{calculated} \mathrm{from}\left(\mathrm{DA}_{\mathrm{A}} / \mathrm{me}_{\mathrm{e}}\right)^{1 / 2}$ if we use values of $\gamma$ from [16]. The maximum arc temperature and properties of the arc at the cathode are largely unaffected by the mesh size at the anode since the plasma flow is directed towards the anode. The arc voltage is, however, influenced by the anode sheath and is reduced by about a volt when the anode mesh size increases from 0.01 to $0.05 \mathrm{~cm}$.

Fig. 4 shows calculated sheath properties at the anode [3] for a $200 \mathrm{~A}$ arc, taking account of space charge effects, through a solution of equations (10) - (13), discussed in the next section. The electric field in the sheath is reversed, producing a negative anode fall, and electron and ion densities are equal to within a few percent to distances of $0.0002 \mathrm{~cm}$ of the anode, which is much less than the electron mean free path.

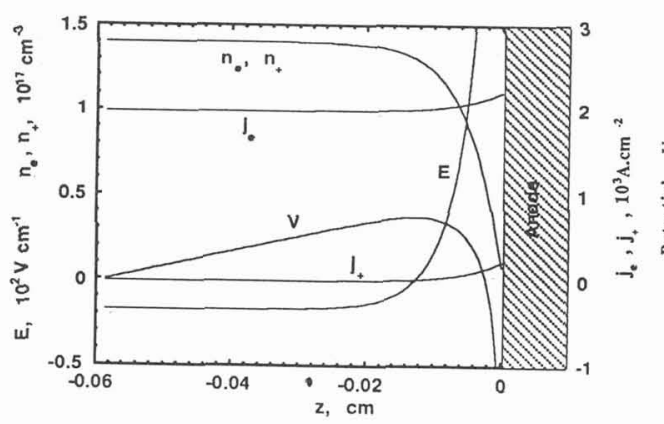

Fig. 4 Derived anode sheath properties for a $200 \mathrm{~A}$ arc [3].

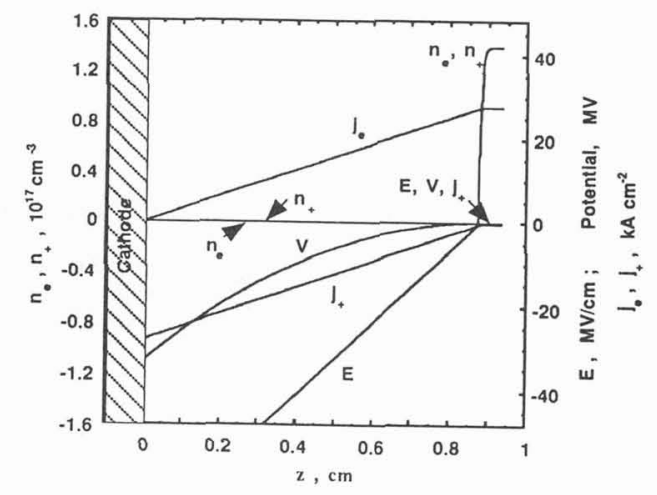

Fig. 5 Derived cathode sheath properties for $S=2 \times 10^{23}$ [3].

\section{NONTHERMIONIC CATHODES AND CATHODE SHEATHS}

For nonthermionic cathodes there is little doubt that space charge effects in the cathode sheath are significant. The change in the electric field from these effects is determined by Poisson's equation.

$$
\frac{d E}{d z}=\frac{e}{\varepsilon}\left(n_{i}-n_{e}\right)
$$

where $\varepsilon=8.85 \times 10^{-14} \mathrm{C} \mathrm{V}^{-1} \mathrm{~cm}^{-1}$ is the permittivity of free space. The voltage, $\mathrm{V}$, across the sheath is obtained by integrating the electric field. Calculations of sheath properties using transport coefficients have recently been made by Lowke and Quartel [3]. For non-thermionic cathodes there is a major question as to how the electrons are produced at the cathode for the arc. The mechanisms of (a) field emission, (b) electron impact ionisation, (c) thermal ionisation and (d) photo-ionisation by ion radiation, are now discussed in turn.

(a) Field emission. The usual view is that electrons are drawn from the cathode by field emission [5], as is generally accepted for vacuum arcs [6]. Electrons moving away from the cathode and positive ions moving toward the cathode produce a layer of high density positive ions next to the cathode which produces a high electric field. The electric field, $\mathrm{E}_{S}$, necessary for field emission is generally taken to 
be $4 \times 10^{7} / \mathrm{cm}$ for a typical work function of $4.5 \mathrm{eV}$ [22]. Typical cathode sheath voltages, $\mathrm{V}_{\mathrm{s}}$, are 20 $V$ or less [4]. By integration of equation (10) twice, neglecting the term in $n_{e}$, we obtain $\dot{E}_{S}=e_{i} b / \varepsilon$, and $V_{S}=$ eni $b^{2} / 2 \varepsilon$, where $b$ is the sheath thickness. Then using the values of $E_{S}=4 \times 10^{7} \mathrm{~V} / \mathrm{cm}$ and $\mathrm{V}_{\mathrm{S}}=20 \mathrm{~V}$, we obtain $\mathrm{b}=10^{-6} \mathrm{~cm}$ and $\mathrm{n}_{\mathrm{i}}=4 \times 10^{19} / \mathrm{cm}^{3}$. Considering that the equilibrium ion density in the arc is approximately $10^{17} / \mathrm{cm}^{3}$ and that ions will be accelerated and have a lower density on approaching the cathode, densities of $4 \times 10^{19} / \mathrm{cm}^{3}$ to produce field emission seem most unlikely. Studies to obtain consistency with fields for field emission and experimental sheath voltages have also been made by other workers [23-24].

(b) Electron impact ionisation. A further possibility is that the electrons are produced by ionisation from electrons accelerated by the high electric field in the sheath. But the secondary-emission coefficient for the production of initiating electrons at the cathode surface is only of the order of 0.1 . Electron currents can at the very most be doubled for a voltage drop in the sheath equal to the ionisation potential. With a secondary emission coefficient of 0.1 we need a doubling of more than 3 times, which would imply sheath voltages of 50 volts or more for argon, where the ionisation potential is $15.8 \mathrm{~V}$.

(c) Thermal ionisation. A further possibility is that electrons are produced by thermal ionisation in the sheath of neutral atoms. These atoms would be brought to a very high temperatures because of the proximity of the arc plasma. Such thermal ionisation must exist in the arc, to balance electron ion recombination, and is represented by a source term, $S$, just as in equation (8). For the general case where electron and ion densities may not be equal, the electron and ion continuity equations are given by

$$
\frac{d j_{e}}{d z}=e S-e \gamma n_{e} n_{i} \quad \text { and } \quad \frac{d j_{i}}{d z}=e S-e \gamma n_{e} n_{i} .
$$

In our calculations we have determined $S$ using $n_{e q}=1.4 \times 10^{17} \mathrm{~cm}^{-3}$, which is the equilibrium electron density for argon at $15000 \mathrm{~K}$ at 1 bar. An approximate estimate of the minimum sheath thickness, $b$, can be obtained by integration of equations (11) to obtain $b=j \mathrm{je} / \mathrm{eS}$, assuming a constant value of $S$ for the sheath. The term in $\gamma$ is neglected because values of $n_{e}$ will be small in the sheath because of the high electron mobility. Furthermore, inclusion of the term in $\gamma$ increases the derived value

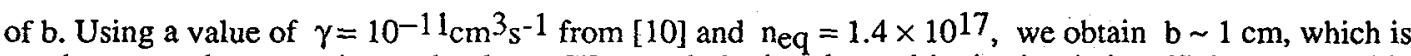
much greater than experimental values. We conclude that thermal ionisation is insufficient to provide the required electrons.

Complete solutions for $n_{e}, n_{i}, E, j e$, and $j_{i}$ for the sheath for the above parameters were obtained in [3] by solving equations (10) and (11) and in addition equations (12) and (13) for the electron and ion current densities;

$$
\begin{gathered}
j_{e}=-e n_{e} \mu_{e} E-e D_{e} \frac{d n_{e}}{d z} \\
j i=-e n_{i} \mu_{i} E .
\end{gathered}
$$

Such a solution, given in Figure 5. All of the spatial derivatives of the five basic variables je, $j_{i}, n_{e}, n_{i}$ and $\mathrm{E}$, are set to zero in the plasma and solutions are obtained for a given value of $j$ by integration of the simultaneous equations (10) - (13) from equilibrium values in the plasma to the cathode. The solution of Figure 5 is unrealistic as the sheath thickness is $\sim 1 \mathrm{~cm}$, in agreement with the approximate calculation.

(d) Photo-ionisation by ion radiation. It is possible that electrons could be produced by photionisation of neutral atoms in front of the cathode by ultra violet radiation from ions in the plasma [3]. Neutral atoms will exist at the cathode surface from the neutralisation of positive argon ions impinging on the cathode and also from background neutral gas. Ultra violet radiation from the equilibrium arc plasma is insufficient to produce the required source electrons as the total emission coefficient for argon at 1 bar and $20000 \mathrm{~K}$ and a thickness of $1 \mathrm{~mm}$ is $10^{3} \mathrm{~W} \mathrm{~cm}^{-3}$ ster $^{-1}$ [27]. Then, for a $1 \mathrm{~mm}$ thick plasma and an ionisation potential of $10 \mathrm{~V}$, there is only a sufficient energy flux to produce an electron current density of $10 \mathrm{~A} / \mathrm{cm}^{2}$, whereas experimental cathode current densities are $\sim 10^{4} \mathrm{~A} / \mathrm{cm}^{2}$.

However, electrons produced by photo-ionisation of neutral atoms at the cathode will be accelerated across the cathode sheath to have an energy equivalent to the sheath voltage, as it is anticipated that the sheath thickness will be of the order of an electron mean free path so that transit across the sheath will be collisionless. Such electrons can excite ions which then radiate. The threshold potential to excite 
radiation from ions is $\sim 18 \mathrm{~V}$, so that the sheath voltage is sufficient for the excitation of radiation. The sum of the cross-sections for the first 5 radiation levels for argon ions, all with thresh-holds of $\sim 18 \mathrm{~V}$, is of the order of $0.2 \times 10^{-16} \mathrm{~cm}^{2}$ [25], so that for ion densities in the plasma of $2 \times 10^{17} \mathrm{~cm}^{-3}$, the absorption distance of the electrons will be $\sim 0.2 \mathrm{~cm}$. This radiation has photon energies greater than the ionisation thresh-hold of $16 \mathrm{~V}$ of neutral atoms, so that it will cause photo-ionisation of the atoms at the cathode. The absorption distance of the radiation is small, ie $-0.03 \mathrm{~cm}$ for a neutral density of $10^{18} \mathrm{~cm}^{-3}$ as the photo-absorption cross-section in argon at this electron energy is $\sim 0.3 \times 10^{-16} \mathrm{~cm}^{2}$ [26]. It is suggested that this photo-ionisation is a principal source of electrons at the cathode for arcs with nonthermionic cathodes.

\section{SPECIFIC PREDICTIONS}

\subsection{Cathode tip melting}

A striking effect occurs with tungsten cathodes having a narrow conical tip, in that at high currents a temperature maximum and melting occurs several $\mathrm{mm}$ from the tip. Thus if the tungsten electrode in "Gas Tungsten Arc Welding" accidentally touches the workpiece, producing high current, the tip of the tungsten can "break" off, as shown in Fig. 6, and become embedded in the weld, forming what is known as a tungsten "inclusion". Systematic surface temperature measurements [28] have shown that there is a maximum in the surface temperature several $\mathrm{mm}$ from the tip, as shown in Figure 7 . Theoretical predictions [11], also shown in Figure 8, also predict a maximum in the surface temperature away from the tip. Thus the tungsten tip actually melts off rather than breaks off. The minimum in the temperature near the tip is due to cooling of the electrode by thermionic emission, and the maximum away from the tip is due to Ohmic heating by the current within the narrow tip of the electrode [11].

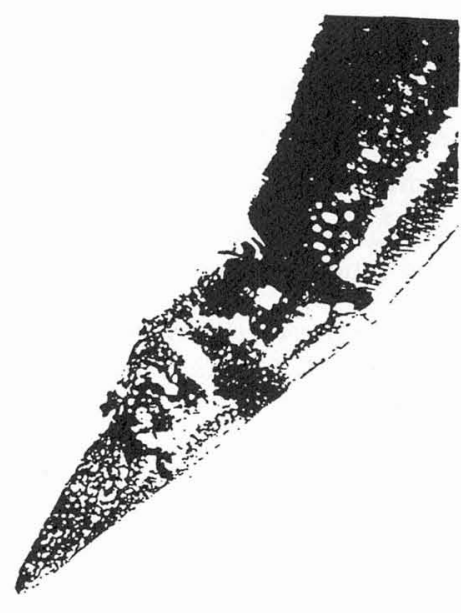

Fig. 6 Tungsten cathode melting away from the tip from tip for $200 \mathrm{~A}$ [28].

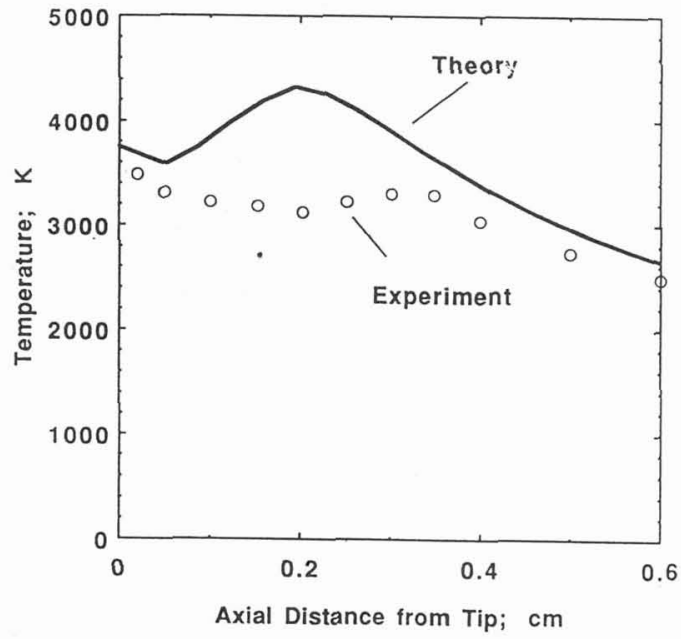

Fig. 7 Theoretical [11] and experimental [28] temperatures at $200 \mathrm{~A}$ for a tungsten cathode of 16 degrees.

\subsection{Effect of $10 \%$ Hydrogen in Argon Arcs}

It is found, in studies related to arc welding [29] that a few percent of hydrogen added to argon causes higher arc temperatures, arc constriction, a higher arc voltage, and a significantly larger volume of molten metal at the workpiece for a given arc current. Such variations can be predicted using the present theory, omitting convection in the molten anode. Figures 8 and 9 show similar calculations to Figure 2 but with an electrode separation of $3 \mathrm{~mm}$ and an anode of mild steel [30]. Figure 8 is for pure argon and Figure 9 is for a mixture of argon and $10 \%$ hydrogen. It is found that the addition of $10 \%$ hydrogen causes a marked increase in the volume of the molten steel, the arc is slightly more constricted and has a slightly increased central temperature and voltage. 
The only difference in the calculations of Figures 8 and 9 are those of the material functions of argon and argon with $10 \%$ hydrogen. These material functions generally differ by only the order of $10 \%$, but with one exception, namely the values of thermal conductivity. At temperatures of around $3500 \mathrm{~K}$, where the dissociation of molecular hydrogen is significant, the thermal conductivity is almost a factor of 10 higher for the mixture than for pure argon [30]. It is this increased thermal conductivity which causes the increased volume of molten metal for the mixture containing hydrogen. The larger thermal conduction also causes a slightly more constricted arc with a higher central temperature and arc voltage.

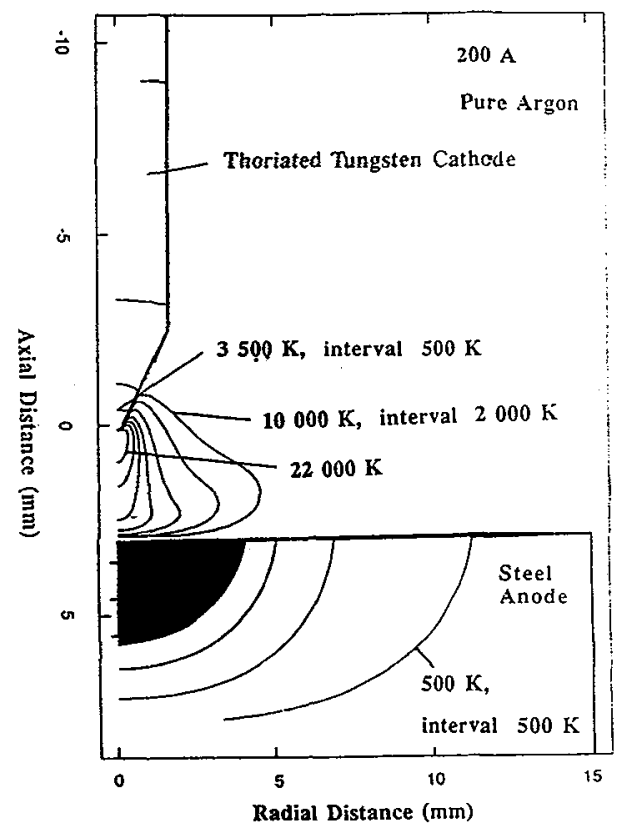

Fig. 8 Calculated Contours, 200A, Ar, steel workpiece, molten volume black [30].

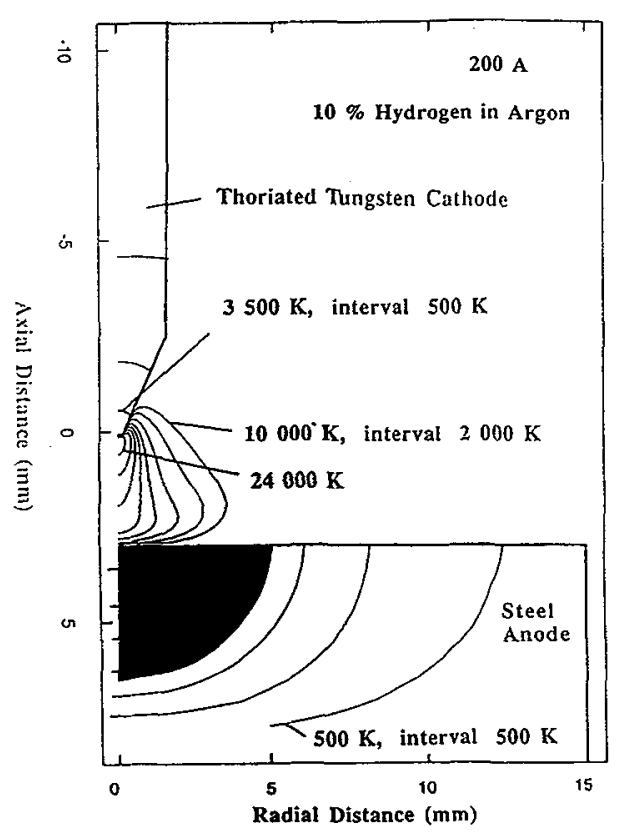

Fig. 9 Calculated contours, $200 \mathrm{~A}, \mathrm{Ar}+10 \% \mathrm{H}_{2}$ steel workpiece, molten volume black [30].

\section{6,3 Globular and Spray modes in Arc Welding}

One of the most striking properties found experimentally in "Gas Metal Arc Welding", where an arc is struck between the welding wire, as the anode, and the workpiece, as the cathode, is that at low currents the wire melts producing large molten drops or globules which generally have a diameter which is larger than the diameter of the wire. Then, above a discrete current, usually about 300A for an arc in argon with $1.6 \mathrm{~mm}$ diameter wire, there is a sudden mode change in the metal transfer, in that diameter of the drops is then smaller than the diameter of the wire. This mode is described as "spray" transfer; Lancaster [31]. The size of the droplets has a marked influence on the properties of the weld.

These differing modes of metal transfer have been obtained theoretically by Haidar and Lowke [32] using the present theoretical methods. The equations used are the same as equations (1) - (6) except that in addition account must be made of the molten liquid phase. Also the calculations must be time dependent as the arc and electrode properties vary in time as each drop is produced from the wire and is detached into the arc. The effect of surface tension acting in the surface of the liquid is to compress the drop and effectively increase the pressure at the surface of the drop by $P_{S}$, where

$$
P_{S}=\Gamma\left(\frac{1}{R_{1}}+\frac{1}{R_{2}}\right) ;
$$


$\Gamma$ is the surface tension coefficient and $R_{1}$ and $R_{2}$ are principal radii of curvature of the surface [33]. Thus the effect of surface tension is to introduce in equations (3) and (4) a step function increase in the pressure $P$ from the plasma to the liquid at the drop surface of an amount given by $P_{S}$ from equation (14). For a spherical surface of radius $R$, this pressure is $P_{S}=2 \Gamma / R$.

In the theoretical predictions of the time dependence of drop formation, again in a unified arcelectrode treatment, it is necessary to make accurate predictions of the curvature of the droplet surface as a function of time, in order to accurately account for the surface tension pressure from equation (6). Such a treatment is possible using the "Volume of Fluid" method [32]. Figure 10 shows the prediction of a large diameter drop, just before detachment, corresponding to the globular mode, for a current of $275 \mathrm{~A}$ in argon, with a wire diameter of $1.6 \mathrm{~mm}$. Figure 11 shows a small droplet, just before detachment, corresponding to the spray mode, for a current of $325 \mathrm{~A}$, also in argon for a wire diameter of $1.6 \mathrm{~mm}$. For currents near the transition current, the calculations usually give a mixture of small and large drops, occurring at different times [32].

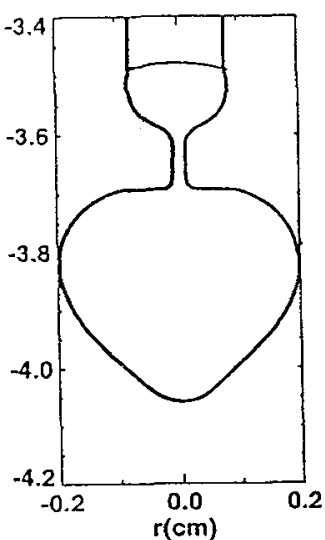

Fig. 10 Calculated drop,

$275 \mathrm{~A}, 1.6 \mathrm{~mm}$ wire [32]

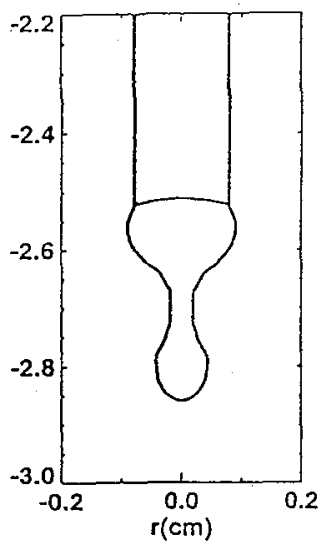

Fig. 11 Calculated drop $325 \mathrm{~A}, 1.6 \mathrm{~mm}$ wire $[32]$.

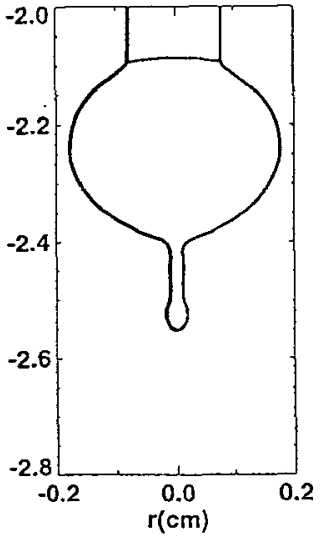

Fig. 13 Calculated drop development, Carbon dioxide, $325 \mathrm{~A}$ [40].

From the detailed calculations of droplet formation of Figures 10 and 11 it is possible to assess the order of magnitude of the various physical forces influencing drop detachment. The force of surface tension tends to compress the drop and hold it on to the wire. At low currents, magnetic pinch forces due to the self magnetic field of the arc are small and drop sizes grow until gravitational forces overcome the surface tension force tending to hold the drop on to the wire. These drop sizes are larger in diameter than that of the wire, as in globular transfer.

The self magnetic field from the current in the drop exerts a pinch force which tends to increase the pressure inside of the drop. For current, I, flowing in a uniform cylinder, of radius R, Maecker [34] showed that this magnetic pinch force increases the effective pressure on the axis of the cylinder by an amount $P_{m}$, where $P_{m}=\mu I^{2} / 4 \pi^{2} R^{2} ; \mu=4 \pi 10^{-7} N / A^{2}$ is the permeability. This pressure increase will tend to extrude liquid from the base of the drop if it is greater than the pressure of $2 \gamma / \mathrm{R}$ from surface tension holding the hemisphere to the solid wire. As the current increases the magnetic pinch pressure increases so that there will be a critical current given by $\mu I^{2} / 4 \pi^{2} R^{2}=2 \Gamma / R$ beyond which surface tension will no longer be able to support the drop of radius equal to the wire radius. Thus an approximate formula for the critical current for transition from the globular mode of transfer to the spray mode of transfer is given by

$$
\mathrm{I}=2 \pi(2 \Gamma \mathrm{R} / \mu)^{1 / 2}
$$

The above derivation is highly approximate in that the effects of gravity, viscous drag forces of the gas surrounding the drop and the pressure of the arc on the drop are all neglected. Figure 12 shows curves of theoretical predictions from Equation (15) for the transition current as a function of wire 
diameter for mild steel and aluminium. The curves marked 'Overhead' and 'Normal' were obtained by including a term $\rho g$ for gravity, in the derivation. For steel the value of $\Gamma$ was taken as $1.2 \mathrm{~N} / \mathrm{m}$. For aluminium the value of $\Gamma$ was taken as $0.28 \mathrm{~N} / \mathrm{m}$. Experimental values for the transition current are shown as points from references [35 - 37] for steel and [38] for aluminium. The values of $\Gamma$ that have been used have been taken for the boiling point of these metals from Smithell's Metals Reference Book [39]. It is seen that despite the approximations made in the theory, agreement between theory and experiment is very good. The approximate physical model used to derive equation (15), treated more thoroughly in [40], provides a simple physical picture of the reason for the onset of the small droplet mode of spray transfer in GMAW. For arcs in carbon dioxide, however, the simple physical picture at the basis of equation (7) does not apply. Arcs in carbon dioxide are much more constricted than arcs in pure argon and as a consequence the pressure of the arc on the drop is much larger. As a result drop development is different than in argon, as is discussed in the next section.

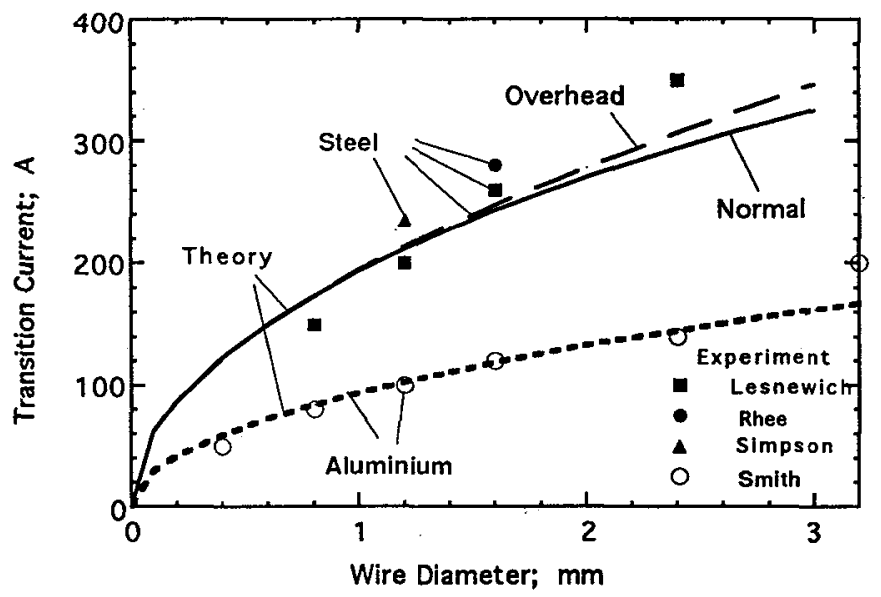

Figure 12: Comparison of experimental results of transition current for spray transfer with predictions from the approximate formula [40].

\subsection{Electrode Melting in Carbon Dioxide Arcs}

It is well known that if arc welding is attempted using pure carbon dioxide gas, there is very different metal droplet behaviour compared with welding in argon [31]. In particular there is much "spatter" of molten electrode material and frequently metal droplets are repelled away from the workpiece and welding wire in a direction counter to the imposed gas flow towards the work piece.

When we make predictions of arc-electrode behaviour, similar to those in argon shown in Figures 2, 8 and 9 , we obtain markedly different arc and droplet behaviour [41]. The central arc temperature for an arc current of $300 \mathrm{~A}$ is $\sim 30000 \mathrm{~K}$ in carbon dioxide compared with a temperature of $24000 \mathrm{~K}$ for argon and the arc is much more constricted in carbon dioxide. Instead of there being fairly distinct globular and spray modes of droplet behaviour, as shown for argon in Figures 10 and 11, in carbon dioxide we generally obtain both modes together, with very large drops, separated by the production of hundreds of very much smaller drops, as is shown in Fig. 13. The higher current density for arcs in carbon dioxide, produces a high arc pressure at the base of the drop, which for the very large drops can be larger than the pressure inside of the drop, tending to repel the drop towards the electrode. Of course our calculations [41] impose two dimensional symmetry, so that the "repelled transfer" mode of drop behaviour often observed in welding with high carbon dioxide concentrations, cannot be simulated theoretically. 


\section{Acknowledgments}

The author is most indebted to his colleagues and coworkers of the CSIRO Division of Telecommunications and Industrial Physics, namely Jawad Haidar, Dick Morrow, Tony Farmer, Gerry Haddad, and Tony Murphy, on whose work the present paper depends.

\section{References}

[1] Lowke J. J., Kovitya P. and Schmidt H. P. J. Phys.D:Appl. Phys. 25 (1992) 1600-1606.

[2] Zhu P., Lowke J. J. and Morrow R. J. Phys.D:Appl. Phys. 25 (1992) 1221-1230.

[3] Lowke J. J. and Quartel J. C. Aust. J. Phys. 50 (1997) 539-552.

[4] Kesaev I. G. Soviet Physics-Technical Physics 9 (1965) 1146-1154.

[5] Yokomizu Y., Matsumura T., Henmi R. and Kito Y. J. Phys. D: Appl. Phys. 29 (1996) 1260- 1267.

[6] Lafferty J. M. Vacuum Arcs, Theory and Application, (Wiley, New York, 1980).

[7] Finkelnburg W. and Maecker H., Handbuch der Physik 22 (1956) 254-444.

[8] Winters H. F., Coufal H., Rettner C. T. and Bethune D. S. Phys.Rev. B 41 (1990) 6240-6256.

[9] Morrow R. and Lowke J. J. J. Phys. D: Appl.Phys. 26 (1993) 634-642.

[10] Zhu P., Lowke J. J., Morrow R., and Haidar J., J. Phys. D:Appl. Phys. 28 (1995) 1369-1376.

[11] Lowke J. J. Morrow R. and Haidar J. J. Phys. D:Appl. Phys. accepted for publication.

[12] Cobine J. (1958). Gaseous Conductors, (Dover, New York, 1958).

[13] Hinnov E and Hirschberg J. G. Phys. Rev. 125 (1962) 795-801.

[14] Mitchner M. and Kruger C. H. Jr. Partially lonized Gases (Wiley, New York, 1973).

[15] Biberman L. M., Vorob'ev V. S. and Yakubov I. T. Soviet Physics Uspekhi 15 (1973) 375-394.

[16] Hoffert M. and Lien H. Phys. Fluids 10 (1967) 1769-1777.

[17] Murphy A. B. and Arundell C. J. Plasma Chem. Plasma Processing 14 (1994) 451-490.

[18] Haddad G. N. and Farmer A. J. D., J. Phys.D:Appl. Phys. 17 (1984) 1189-1196.

[19] Haidar J. and Farmer A. J. D., Rev. Sci. Instrum. 64 (1993) 542-547.

[20] Dinulescu H. A. and Pfender E. J.Appl.Phys. 51 (1980) 3149-3157.

[21] Nestor O. H. J. Appl. Phys. 33 (1962) 1638-1648.

[22] Coulombe S. and Meunier J. L. J. Phys.D:Appl. Phys. 30 (1997) 776-780.

[23] Zhou X. and Heberlein, J. Plasma Sources Sc. Technol. 3 (1994) 564-574.

[24] Benilov M. S. and A. Marotta A. J. Phys.D:Appl. Phys. 28 (1995) 1869-1882.

[25] Zapesochnyi I. P., Imre A. I., Dashchenko A. I., Vukstich V. S., Danch F. F., and Kel'man V. A. Soviet Physics JETP 36 (1973) 1056-1060.

[26] Ditchburn R. W. J. Quant. Spectrosc. Radiative Transfer 2 (1962) 361-368.

[27] Boulos M. I., Fauchais, P. and Pfender, E. Thermal Plasmas: Fundamentals and Applications (Plenum Press, New York, 1994).

[28] Haidar J. and Farmer A. J. D. J. Phys. D:Appl. Phys. 27 (1994) 555-560.

[29] Hoojmans J. W. Hydrogen absorption in iron and steel during gas tungsten arc welding, Thesis, Delft University of Technology, Delft, (1994).

[30] Lowke J. J., Morrow R., Haidar J. and Murphy A. B. IEEE Trans. Plasma Science. Accepted for publication.

[31] Lancaster, J. F.,The Physics of Welding, (Pergamon Press , London, 1984), p 234.

[32] Haidar J. and Lowke J. J. J. Phys.D:Appl. Phys. 29 (1996) 2951-2960 and J. High Temp. Chem. Process. Accepted for publication.

[33] Starling S. G. and Woodall A. J., Pysics, (Longmans, London, 1958), p104.

[34] Maecker H., Z. Phys. 141 (1955) 198-216.

[35] Lesnewitch A., Welding Journal, 37 (1958) 418s-425s.

[36] Rhee R. and Kannatey-Asibu E., Welding Journal, 70 (1992) 381s-386s.

[37] Simpson S. I., Zhu P., and Rados M., Proc. 42nd National Welding Conference, Welding Technology Inst. Australia, Melbourne, 2 (1994) p. 31.1.

[38] Smith A. A. and Poley J. G. British Welding Journal, 6 (1959) 565-568.

[39] Brandes E. A., Smithell's Metals Reference Book, 6th Edition, (Butterworths, London, 1983).

[40] Lowke J. J., Australasian Welding Journal, 42 (1997) 1, 32-35.

[41] Haidar J. and Lowke J. J. IEEE Trans. Plasma Science. Accepted for publication. 